\title{
Geospatial analyses to identify clusters of adverse antenatal factors for targeted interventions
}

\author{
Shanley Chong ${ }^{1,2^{*}}$, Michael Nelson ${ }^{3}$, Roy Byun ${ }^{1}$, Liz Harris ${ }^{4}$, John Eastwood ${ }^{5,6,7,8}$ and Bin Jalaludin ${ }^{1,6}$
}

\begin{abstract}
Background: Late antenatal care and smoking during pregnancy are two important factors that are amenable to intervention. Despite the adverse health impacts of smoking during pregnancy and the health benefits of early first antenatal visit on both the mother and the unborn child, substantial proportions of women still smoke during pregnancy or have their first antenatal visit after 10 weeks gestation. This study was undertaken to assess the usefulness of geospatial methods in identifying communities at high risk of smoking during pregnancy and timing of the first antenatal visit, for which targeted interventions may be warranted, and more importantly, feasible.

Methods: The Perinatal Data Collection, from 1999 to 2008 for south-western Sydney, were obtained from the New South Wales Ministry of Health. Maternal addresses at the time of delivery were georeferenced. A spatial scan statistic implemented in SaTScan was then used to identify statistically significant spatial clusters of women who smoked during pregnancy or women whose first antenatal care visit occurred at or after 10 weeks of pregnancy.

Results: Four spatial clusters of maternal smoking during pregnancy and four spatial clusters of first antenatal visit occurring at or after 10 weeks were identified in our analyses. In the maternal smoking during pregnancy clusters, higher proportions of mothers, were aged less than 35 years, had their first antenatal visit at or after 10 weeks and a lower proportion of mothers were primiparous. For the clusters of increased risk of late first antenatal visit at or after 10 weeks of gestation, a higher proportion of mothers lived in the most disadvantaged areas and a lower proportion of mothers were primiparous.
\end{abstract}

Conclusion: The application of spatial analyses provides a means to identify spatial clusters of antenatal risk factors and to investigate the associated socio-demographic characteristics of the clusters.

Keywords: Antenatal, Pregnancy, Smoking, SaTScan, Geospatial

\section{Introduction}

There are many established risk factors for adverse perinatal outcomes. These include late antenatal care, smoking during pregnancy, maternal infection [1], maternal hypertension [2], gestational diabetes [3] and social factors such as teen pregnancy [4] and lower socioeconomic status [5]. Late antenatal care and smoking during pregnancy are two important factors that are amenable to intervention.

Australia's and UK's NICE antenatal care guidelines recommend that the first antenatal visit should be before

\footnotetext{
* Correspondence: Shanley.chong@sswahs.nsw.gov.au

${ }^{1}$ Centre for Research, Evidence Management and Surveillance, South Western Sydney Local Health Districts, Sydney, Australia

${ }^{2}$ South Western Sydney Clinical School, University of New South Wales,

Sydney, Australia

Full list of author information is available at the end of the article
}

10 weeks of gestation [6,7]. Early first prenatal care is important for the detection of adverse pregnancy related outcomes and is vital for healthy perinatal outcomes in both mothers and infants [8-10]. The benefits include healthy birth weight [11-13], low risk of preterm delivery $[12,14]$ and lower neonatal mortality $[12,14]$. Antenatal care programs not only monitor both maternal and foetal health, but also facilitate health promoting advice such as smoking cessation. Women of low socioeconomic status [15,16], younger women [15], primiparous women $[17,18]$ and indigenous women [19] are associated with late antenatal care.

Smoking during pregnancy is associated with a high risk of miscarriage, stillbirth and serious complications during delivery [20], all of which add to the overall health costs [21]. Younger women are more likely to

\section{Biomed Central}

(c) 2013 Chong et al.; licensee BioMed Central Ltd. This is an open access article distributed under the terms of the Creative Commons Attribution License (http://creativecommons.org/licenses/by/2.0), which permits unrestricted use, distribution, and reproduction in any medium, provided the original work is properly cited. 
smoke during pregnancy [22] and mothers of lower socio-economic status, who were primiparous and attended first antenatal care late were less likely to quit smoking during pregnancy [23].

Despite the negative health impacts of smoking during pregnancy and the health benefits of early first antenatal visit on both mother and unborn child, substantial proportions of women still smoke during pregnancy or have their first antenatal visit after 10 weeks gestation. For example, 20\% of mothers who gave birth during 2002 to 2004 smoked during pregnancy in Western Australia [24] and in New South Wales (NSW), 41\% of women had their first antenatal care late in their pregnancies [17]. Therefore, it is important for health care providers to ensure that both the care providers and the community are aware of the benefits of early prenatal care and the detrimental effects of smoking during pregnancy on maternal and infant health.

Although targeted preventative programs such as a home visiting program can improve maternal healthrelated behaviours during and after pregnancy [25,26], it can be difficult to direct interventions to individual mothers at greatest need. The identification of small geographical areas with a high prevalence of poor maternalrelated behaviour could allow for more targeted efforts at those most at risk.

The application of spatial statistics and geographic information system (GIS) to health outcomes is now being increasingly used to provide novel ways of examining disease patterns geographically [27-29]. SaTScan is a software for implementing spatial, temporal and spacetime scan statistics that can be used to determine areas where an event of interest, for example, cancer incidence or preterm deliveries, appears inconsistent with the overall study area. This technique is not limited to existing administrative boundaries such as postal area, and can identify location of the clusters without a priori knowledge about their location and size [30].

To our knowledge, SaTScan has not been used for analysing geographical patterns of antenatal maternalrelated risk factors. This study was undertaken to assess the usefulness of such geospatial methods in identifying communities at high risk of poor maternal-related behaviours, particularly, smoking during pregnancy and timing of the first antenatal visit, for which targeted interventions may be warranted and importantly feasible.

\section{Methods}

The study area was the south-western region of metropolitan Sydney, Australia. It is about 6382 square kilometres in area, consists of 15 local government areas (LGAs) and had a population of 1,460,847 in the 2011 Census [31]. The population in the 15 LGAs ranged from 32,423 to 187,766 persons, and the population density ranged from 17 to 6,349 persons per square kilometre. In the same census, Metropolitan Sydney covers about 12,368 square kilometres and had a population of 4,391,674 persons [31].

\section{Data source}

The Perinatal Data Collection (MDC), from 1999 to 2008 for south-western Sydney, was obtained from the New South Wales (NSW) Ministry of Health. It is a population-based surveillance system comprising of all births in NSW public hospitals, private hospitals and homebirths. It includes all livebirths and still births of at least 20 weeks gestation or at least 400 grams birth weight. A notification form is completed by the attending midwife for every birth. Information is collected on demographic items and items on maternal health, pregnancy, labour, delivery and the newborn and includes maternal age, smoking during pregnancy (yes/no), timing of first antenatal visit, birth weight, gestational age, single or multiple births, parity and country of birth.

Using the Australian antenatal guidelines, timing of the first antenatal visit was categorised into two groups: first antenatal visit $<10$ weeks and first antenatal visit $\geq 10$ weeks (late first antenatal visit) [6,7].

The 2001 and 2006 Index of Relative Socio-Economic Disadvantage (IRSED) at the census collection district (CCD) level were used in the analyses as an ecological measure of area deprivation [32]. A CCD consists of about 220 households in urban areas and fewer households in rural and semi-rural areas. The IRSED at the CCD level was created by the Australian Bureau of Statistics to compare social and economic disadvantage across geographical areas in Australia. The index is derived from Census variables such as low income and educational attainment, high unemployment, and people working in unskilled occupations. The index has a mean score of 1,000 and standard deviation of 100 [33]. The IRSED was categorised into quintiles. Quintile 1 is designated as most disadvantaged, quintile 2 to quintile 4 are combined and designated as the middle disadvantaged group and quintile 5 is designated as the least disadvantaged group. All women living in a particular CCD were assigned the IRSED for that CCD.

\section{Statistical methods}

Maternal residential addresses at the time of delivery were georeferenced and imported into spatial scan statistic (SaTScan) for analysis. A spatial scan statistic implemented in SaTScan was used to identify the presence of statistically significant spatial clusters of women who smoked during pregnancy or women whose first antenatal care visit occurred $\geq 10$ weeks of pregnancy [34]. The analysis was conducted using a Bernoulli model, binary event data. Women who did not smoke during 
pregnancy or whose first antenatal care occurring before 10 weeks of pregnancy were assigned as controls. A spatial scan statistic uses a scan window (the population at risk) either in the shape of a circle or an ellipse, which moves across the study region [34,35]. We present our results using the ellipse window and a medium strength compactness penalty because it provided higher sensitivity than the circular-shaped window for late antenatal visit (53\% vs $49 \%$, respectively) and similar sensitivity for maternal smoking ( $42 \%$ vs $43 \%$, respectively).

For each location, the size of the scan window varies from 0 to a specified maximum value. For the purposes of this study, the size of the scan window was set to no more than $20 \%$ of the study population, to scan for small clusters which may possibly be more amenable to interventions. For each window, the alternative hypothesis is that there is a difference in the risk of poor maternal health behaviour within the window as opposed to outside the window. The likelihood function is maximised over all windows, and the window with the maximum likelihood constitutes the most likely cluster. We selected the non-overlapping option in SaTScan when generating secondary clusters. Clusters with significant large likelihood ratios are identified. The test of significance of the identified clusters is based on a likelihood ratio test whose $\mathrm{p}$-value is generated by applying Monte Carlo replications $[35,36]$. The number of Monte Carlo replications was set to 999 to ensure adequate power for defining clusters and a p-value less than 0.05 was considered statistically significant.

Using purely spatial scan statistics, we also examined for clusters using two year time periods (1999-2000, 2001-2002, 2003-2004, 2005-2006, 2007-2008). The locations and sizes of the clusters did not vary greatly by these two periods. The spatial-temporal clusters that we detected were similar in locations and size to the spatial only clusters, except for late antenatal visit between 2007 and 2008 where two new clusters were found. These two clusters were also detected using purely spatial scan and space-time scan statistics. This indicates the occurrence of maternal smoking is mainly spatial rather than temporal in our study area. For simplicity,

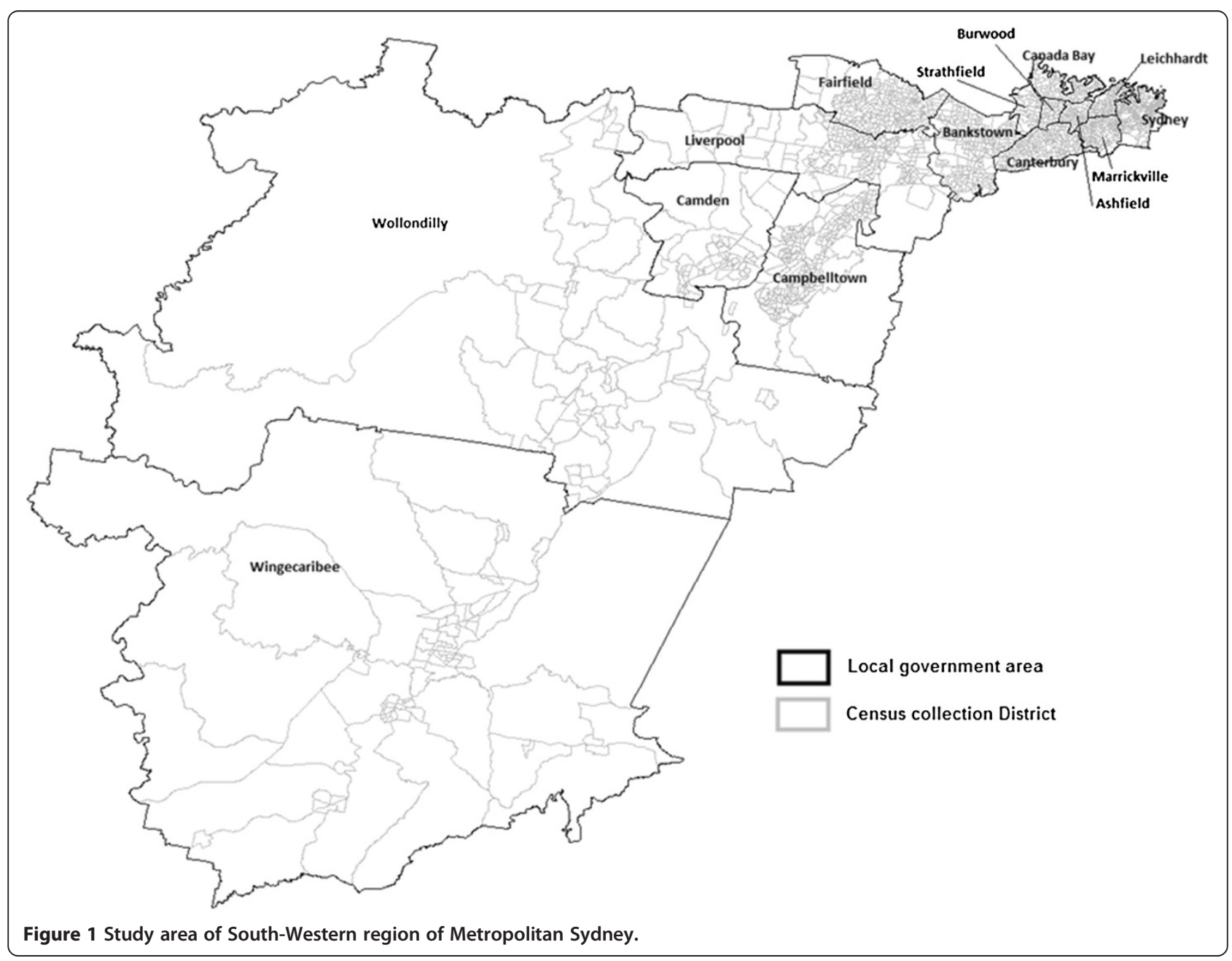


only spatial clusters based on purely spatial scan were presented.

The specific locations of clusters were evaluated in terms of relative risks (RRs). A cluster with a RR of $>1$ indicates an increased risk for that cluster compared to the risk outside that cluster. Kernel density was then used to visually explore the variability of the density over the surface of these clusters into Google map using R-studio [37]. To account for correlation among women within clusters, generalised estimating equations (GEE) logistic regression models with logit link and compound symmetry correlation were used to examine the associations between socio-demographic and clinical characteristics of women inside and outside the significant clusters.

Ethics approval for this study was obtained from the NSW Population \& Health Services Research Ethics Committee.

\section{Results}

Figure 1 shows the study area of south-western region of metropolitan Sydney. From 1999 to 2008, there were 195,500 births in this study area. About three percent of the mothers were aged less than 20 years, $76.6 \%$ were aged between 20 and 34 years and $20.1 \%$ aged 35 years or more. Just over half of the women were born in Australia (54.6\%). About $12 \%$ of women smoked during the pregnancy and $30.7 \%$ of women had their first antenatal visit $\geq 10$ weeks of gestation. The majority of women delivered a singleton infant (97.0\%). Only a small proportion of women had gestational diabetes or gestational hypertension $(6.6 \%$ and $4.5 \%$ respectively (Table 1 ).

\section{Spatial analysis}

Using the maximum spatial circular windows $\leq 20 \%$ of the total population, we identified a number of spatial clusters of women who smoked during pregnancy (Figure 2) and whose initial antenatal visit occurred $\geq 10$ weeks gestation (Figure 3). These clusters are presented in order of the most likely cluster to the least likely cluster.

Four significant clusters of maternal smoking during pregnancy were generated with RRs ranging from 1.22 to 2.66. The most likely cluster covered $12.0 \%(n=23,557)$ of all births and was mainly located in one LGA. The overall RR for this cluster was $2.66(\mathrm{p}<0.01)$. The three secondary clusters contained $12.3 \%$ of all births.

For late first antenatal visit occurring $\geq 10$ weeks gestation, four significant clusters were generated with RRs between 1.03 and 1.10. The most likely cluster covered $18.3 \%(\mathrm{n}=35,834)$ of all births in the study area and was again mainly located in one LGA $(R R=1.10, p<0.001)$. The second and third were mainly located in densely populated areas of two separate LGAs and the fourth
Table 1 Maternal demographic and clinical characteristics in the study area $(\mathrm{N}=195,500)$

\begin{tabular}{|c|c|}
\hline & n (\%) \\
\hline \multicolumn{2}{|l|}{ Age-group } \\
\hline$<20$ year & $6431(3.3)$ \\
\hline $20-<35$ years & $149,762(76.6)$ \\
\hline $35+$ years & $39,307(20.1)$ \\
\hline \multicolumn{2}{|l|}{ Country of birth } \\
\hline Australian & $106,769(54.6)$ \\
\hline Overseas & $88,731(45.4)$ \\
\hline \multicolumn{2}{|l|}{ Index of Relative Socio-economic Disadvantage } \\
\hline \multicolumn{2}{|l|}{ (missing $=1,883)$} \\
\hline Most disadvantage & $64,541(33.3)$ \\
\hline Middle disadvantage and Least disadvantage & $129078(66.7)$ \\
\hline \multicolumn{2}{|l|}{ Number of babies } \\
\hline Multiple & $5,886(3.0)$ \\
\hline Singleton & $189,614(97.0)$ \\
\hline \multicolumn{2}{|l|}{ Primiparous (missing = 448) } \\
\hline Yes & $83,263(42.6)$ \\
\hline No & $111,789(57.2)$ \\
\hline \multicolumn{2}{|l|}{ First antenatal visit (missing $=3,049$ ) } \\
\hline$\geq 10$ weeks & $60,041(30.7)$ \\
\hline$<10$ weeks & $132,410(67.7)$ \\
\hline \multicolumn{2}{|l|}{ Smoking during pregnancy (missing $=1,613$ ) } \\
\hline Yes & $22,635(11.6)$ \\
\hline No & $171,252(87.6)$ \\
\hline
\end{tabular}

cluster covered most of a densely populated area in a LGA, with $12.5 \%, 10.7 \%$ and $7.8 \%$ of all births respectively.

\section{Characteristics of the clusters}

Table 2 shows demographic characteristics for the identified clusters for maternal smoking and the first antenatal visit at or after 10 weeks gestation compared to the rest of the study area. Compared to women in the rest of the study area, women in the identified clusters for maternal smoking during pregnancy $(\mathrm{n}=47,593$ in the significant spatial clusters, $24 \%$ of all births), were aged less than 35 years $(85 \%$ vs $78 \% ; \mathrm{p}<0.0001)$ and have their first antenatal visit at or after 10 weeks $(79 \%$ vs $75 \%, \mathrm{p}=0.011$ ). The clusters also had significantly lower proportions of women who were primiparous (37\% vs 45\%; $\mathrm{p}<0.0001$ ).

In the identified clusters for women who had their first antenatal care $\geq 10$ weeks gestation, there were 96,308 (49\% of all births) women in the three clusters compared to 99,192 women in the remainder of the study area. These clusters had significantly higher proportions of women who lived in the most disadvantaged areas (56\% vs $11 \%, \mathrm{p}<0.0001)$ compared to the rest of the study 


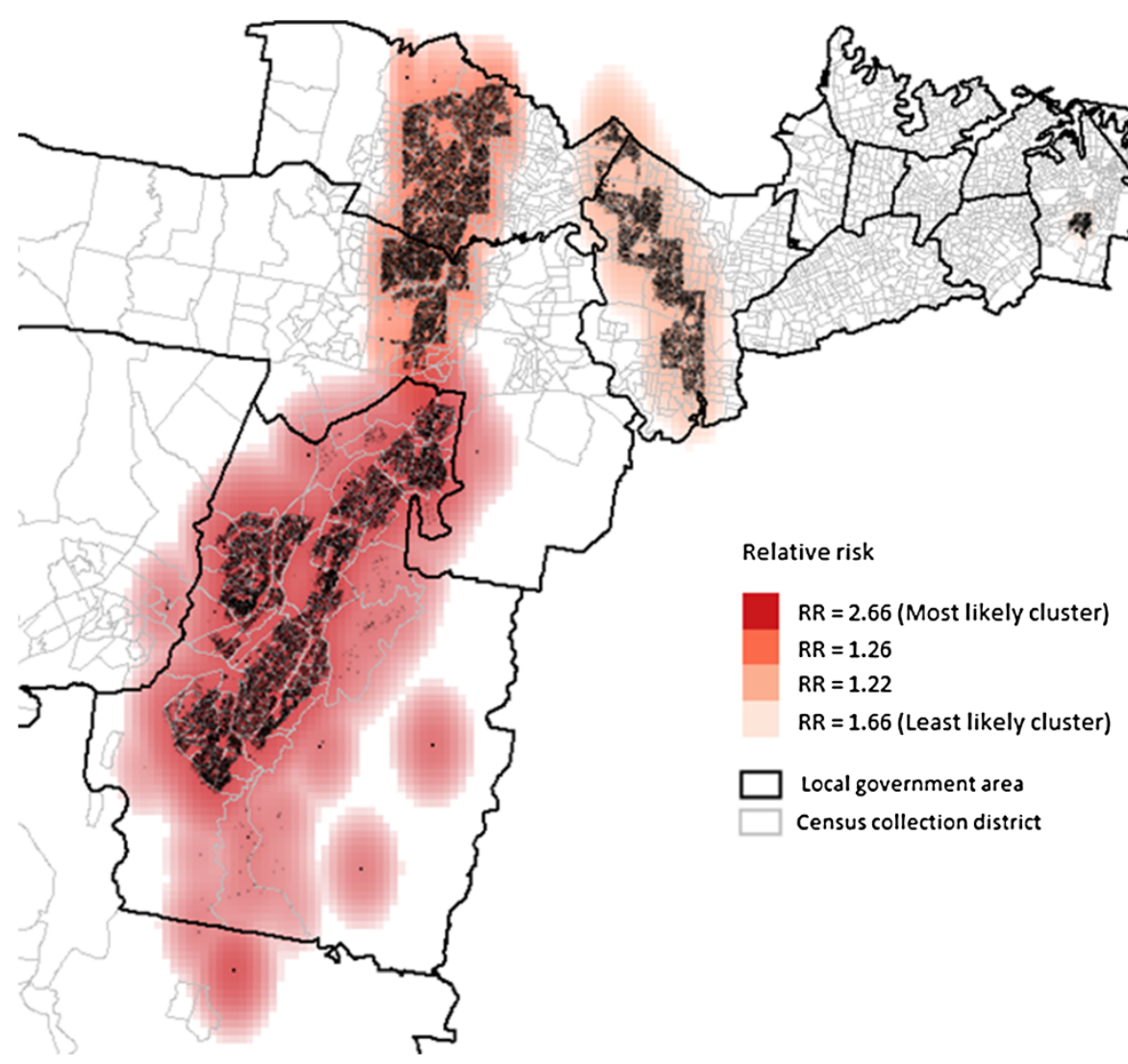

Figure 2 Clusters of maternal smoking during pregnancy using the maximum cluster size $\leq \mathbf{2 0} \%$.

area. These clusters also had lower proportions of women who had multiple births (2.9\% vs $3.2 \%, \mathrm{p}<0.0001)$.

As the significant spatial clusters of the two risk factors overlapped, we also overlaid the clusters of maternal smoking with the clusters of first antenatal visit at or after 10 weeks of gestation to identify significant spatial clusters of both smoking during pregnancy and late antenatal visits (Figure 4). Areas around the green dots in Figure 4 indicate areas where there is a high risk for both maternal smoking and late first antenatal visit at or after 10 weeks of gestation.

Figure 5 shows an additional spatial analysis based on a maximum circular window of $10 \%$ of the total study population. This additional analysis identified four significant spatial clusters as shown in Figure 2 when using a maximum circular window of $20 \%$ of the population. These four spatial clusters are located in the same spatial clusters identified with a maximum circular window of $20 \%$ of the study population (Figure 2), but are smaller in size. The most likely cluster in this additional analysis comprised $9.7 \%(n=19,061)$ of all births in the study area compared to $18.3 \%(n=35,834)$ of births based on a maximum circular window of $20 \%$ of the total study population.

\section{Discussion}

We identified four spatial clusters of maternal smoking during pregnancy and four spatial clusters of first antenatal visit occurring at or after 10 weeks of gestation. Following identification of the clusters, we were also able to characterise pregnant women within the clusters and compare those characteristics with women living outside of those clusters. For example, in the maternal smoking during pregnancy clusters, higher proportions of mothers, were aged less than 35 years, were born in Australia, had their first antenatal visit at or after 10 weeks of gestation and a lower proportion of mothers were primiparous. In the clusters of late first antenatal visit, a higher proportion of mothers lived in the most disadvantaged areas and a lower proportion of mothers were primiparous.

SaTScan is a widely used and accepted software for geospatial analytic technique to identify and describe geographic patterns of interest in the public health literature [38-41]. Our study used both spatial statistics and descriptive statistics to describe adverse maternal-related behaviours in the south western region of metropolitan Sydney. However, it should be noted here that, although we have demonstrated the usefulness of using SatScan to identify the spatial distribution of adverse risk factors, 


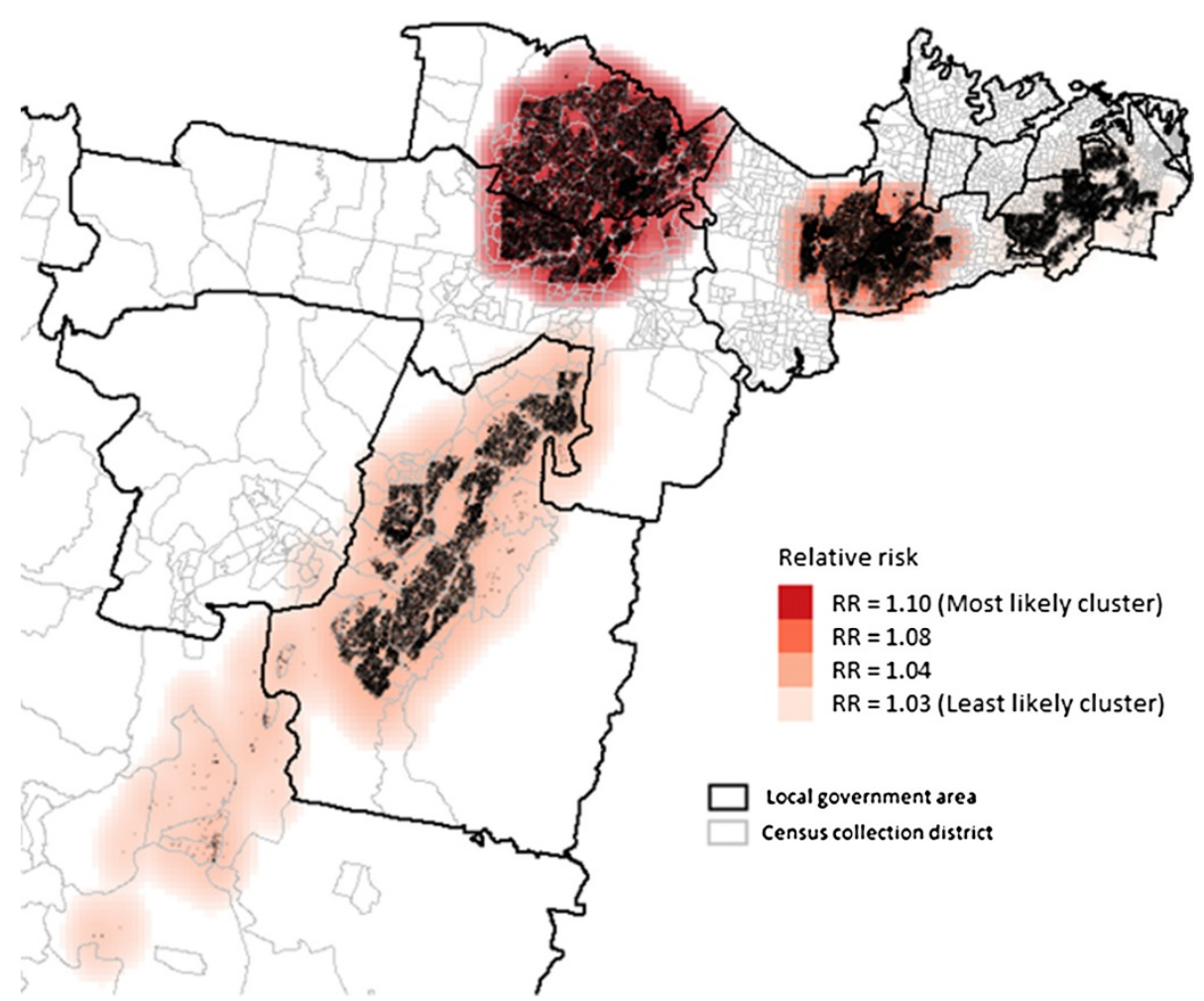

Figure 3 Clusters of first antenatal visit at or after 10 weeks and above using the maximum cluster size $\leq 20 \%$.

SaTScan can also be used to identify spatial distributions favourable risk factors or health outcomes, for example, areas with low rates of smoking or certain cancers. An identified spatial cluster where the causal mechanism is not readily apparent could have merely occurred by chance [42]. Therefore, any identified clusters need to be further investigated and the results and conclusions supported by other published studies [42]. For example our finding of significant differences in risk of smoking during pregnancy across socio-demographic groups is consistent with previous studies [22,23]. Similarly, the identification of differences in risk of late antenatal care across our study area is consistent with previous studies that late antenatal care is related to mother's age [15], area of residence $[15,43]$ and low socio-economic status $[15,16,44]$. This reinforces the robustness of the results obtained from SaTScan.

Demonstrating differences in the socio-demographic factors underlying the identified clusters can assist policy makers in understanding the epidemiology of particular diseases, risk factors or health issues, and to develop and implement interventions and reorient health services. For example, SaTScan has been used to implement antimarlarial interventions at the household level [45], to identify clusters of hypoplastic left heart and assess genetic and environmental factors contributing to hypoplastic left heart [46], and planning regional tuberculosis prevention and control strategies by identifying clusters of high incidence of tuberculosis [47].

Our study, by identifying adverse maternal-related behaviours by spatial clusters or geography, provides valuable information about the geographical disparity of adverse maternal-related behaviour. It also provides additional insights to the characteristics of the cluster that contribute to adverse perinatal outcomes. Local knowledge and understanding of the physical characteristics of the identified geographical areas (for example, availability of public transport, location of health services) and socio-demographic characteristics of the women within the clusters can assist policy makers to focus the scope of prevention/intervention programs and changes to health care delivery, thus providing more effective programs to suit individual needs and public health resources can be delivered more efficiently.

We also overlaid the clusters of maternal smoking with the clusters of first antenatal visit to identify significant spatial clusters of both smoking during pregnancy and late antenatal visits. Therefore, if there are limited resources, targeting initially only those spatial clusters with both risk factors may be a cost-effective approach to improving maternal and birth outcomes.

Public health interventions can be directed at a number of geographic levels. They can be at a national level (for example, smoking cessation campaigns using the 
Table 2 Demographic characteristics for significant spatial cluster compared to the remainder of the study area using GEE logistic regression

\begin{tabular}{|c|c|c|c|c|c|c|}
\hline & \multicolumn{3}{|c|}{ Smoking during pregnancy clusters $\mathrm{n}(\%)$} & \multicolumn{3}{|c|}{ 1st antenatal visit at $\geq 10$ weeks clusters $n(\%)$} \\
\hline & Clusters & $\begin{array}{l}\text { Remainder of } \\
\text { the study area }\end{array}$ & $\overline{p \text {-value }}$ & Clusters & $\begin{array}{l}\text { Remainder of } \\
\text { the study area }\end{array}$ & p-value \\
\hline & 47,593 & 147,907 & & 96,308 & 99,192 & \\
\hline Smoke during pregnancy & $8,767(18.6)$ & $11,768(8.1)$ & $<0.0001$ & $11,703(12.3)$ & $8,832(9.1)$ & 0.2465 \\
\hline 1 st antenatal visit $\geq 10$ weeks & $36,991(79.2)$ & $109,491(75.1)$ & 0.0112 & $77,767(80.8)$ & $68,715(71.5)$ & $<0.0001$ \\
\hline Overseas-born & $19,681(41.4)$ & $69,050(46.7)$ & 0.5045 & $52,914(54.9)$ & $35,817(36.1)$ & 0.0623 \\
\hline \multicolumn{7}{|l|}{ Maternal age (years) } \\
\hline$<35$ & $40,552(85.2)$ & $115,607(78.2)$ & $<0.0001$ & $79,123(82.2)$ & $77,036(77.7)$ & 0.3988 \\
\hline$\geq 35$ & $7,034(14.8)$ & $32,273(21.8)$ & & $17,171(17.8)$ & $22,136(22.3)$ & \\
\hline \multicolumn{7}{|c|}{ Index of Relative Socio-economic Disadvantage } \\
\hline Most disadvantaged & $20,447(44.0)$ & $44,094(30.0)$ & 0.4621 & $56,621(56.4)$ & $10,920(11.1)$ & 0.0014 \\
\hline Middle and least disadvantaged & $26,078(56.1)$ & $103,000(70.0)$ & & $41,437(46.6)$ & $87,641(88.9)$ & \\
\hline Plurality & $1,386(2.9)$ & $4,500(3.0)$ & 0.1162 & $2,748(2.9)$ & $3,138(3.2)$ & 0.0021 \\
\hline Primiparous & $17,616(37.0)$ & $65,647(44.5)$ & $<0.0001$ & $40,000(41.5)$ & $43,263(43.8)$ & 0.8380 \\
\hline
\end{tabular}

mass media) at the one extreme to very local interventions (for example, modifying the physical characteristics of a locality) at the other extreme. Interventions at both these geographic levels can be equally valid and important. SaTScan allows us to vary the size of the scan windows and hence the number and geographic dimensions of the clusters.

Clusters generated using a larger size scan windows produce larger clusters which can help policy makers make decisions at larger geographic levels, for example, at the state of regional level. However, these large spatial clusters will cover a large area with a larger and more heterogeneous population. Conversely, clusters generated using smaller circular windows will produce smaller clusters but will contain a more homogeneous population which can help policy makers in planning more focused community interventions $[48,49]$. For example, Fang et al. used circular windows no more than $20 \%$ to

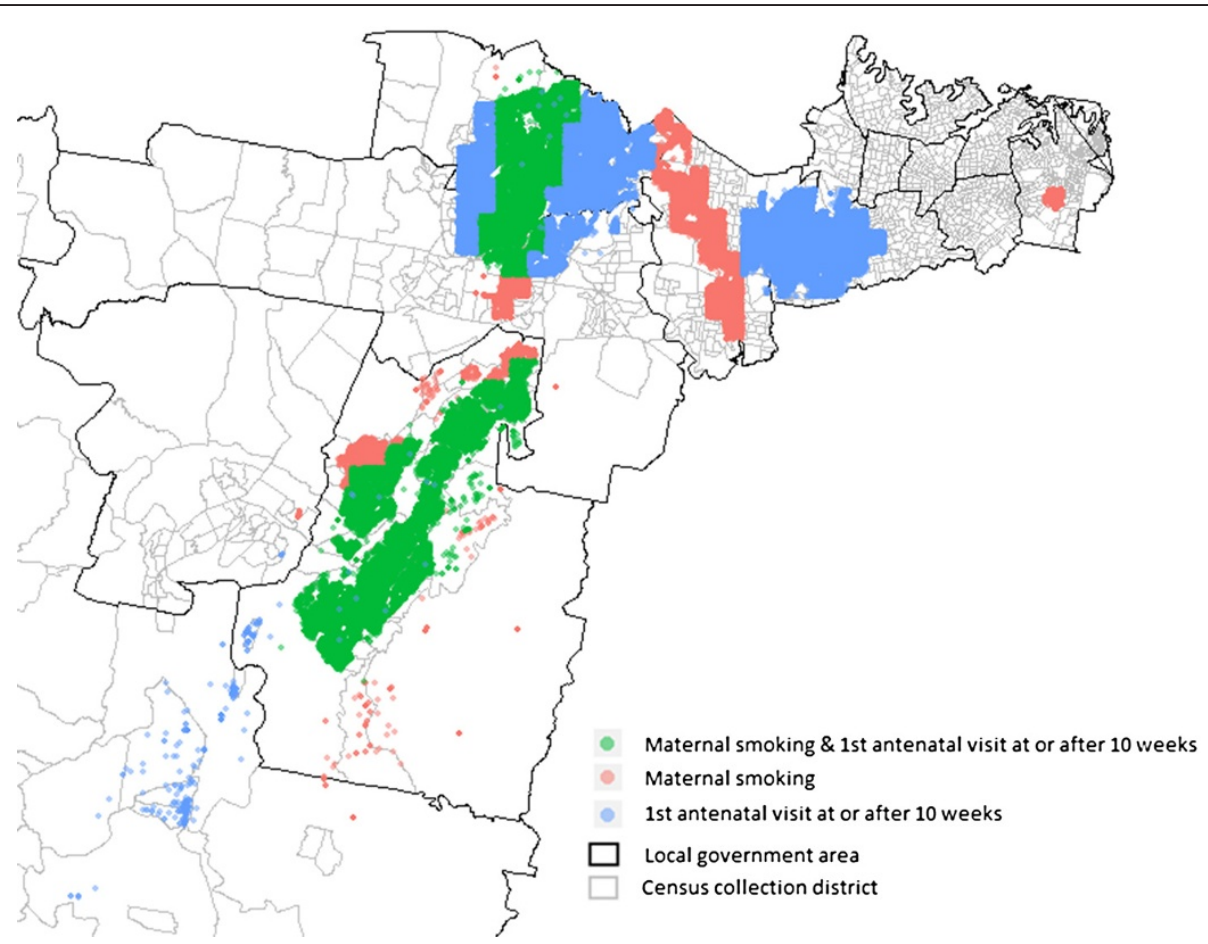

Figure 4 Clusters of maternal smoking overlaid with clusters of first antenatal visit at or after 10 weeks. 


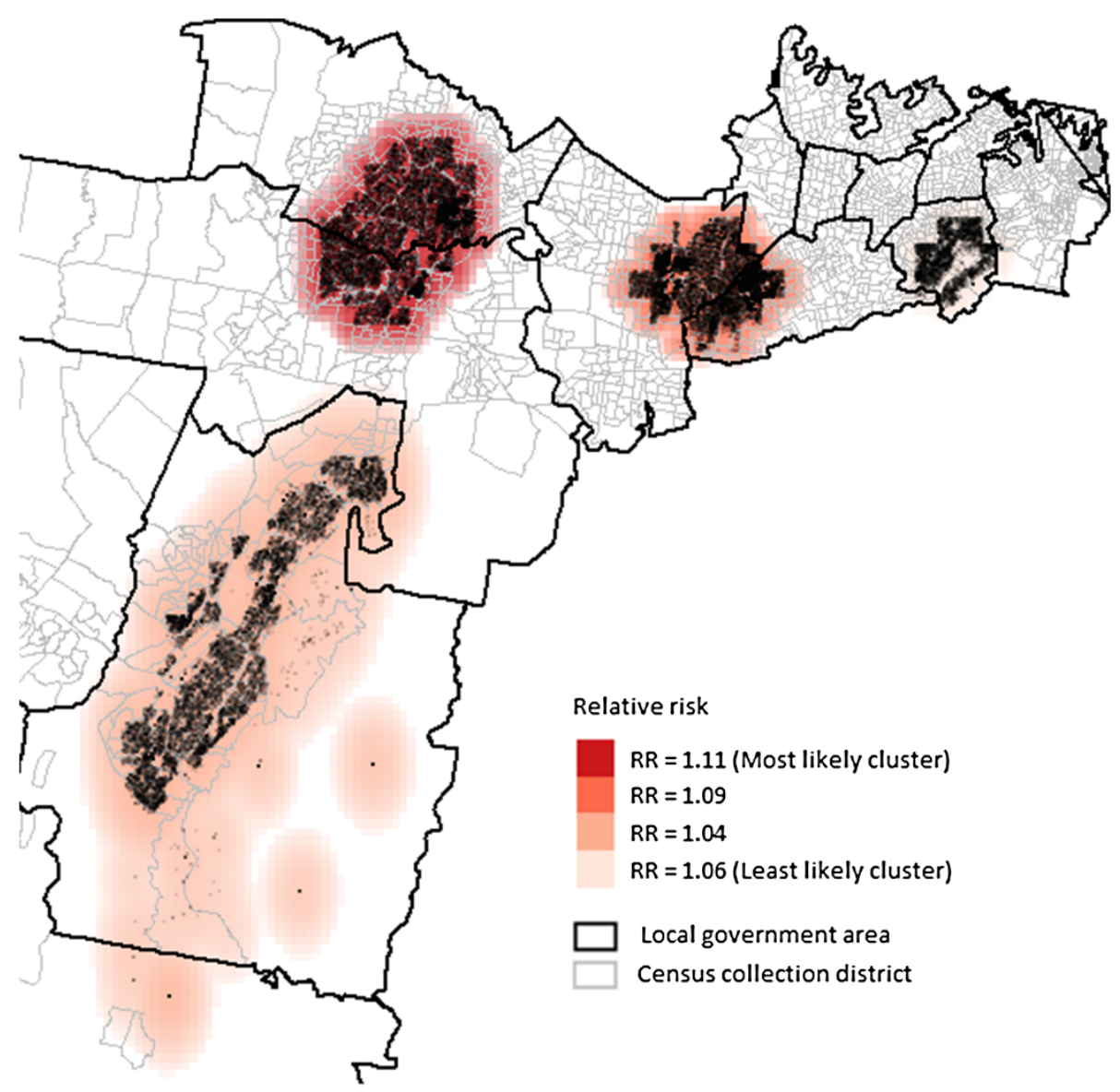

Figure 5 Clusters of first antenatal visit at or after 10 weeks with the maximum cluster size $10 \%$ of the total study population.

identify hemorrhagic fever with renal syndrome clusters and smaller circular windows no more than $10 \%$ to identify possible subclusters for more efficient resource allocation for preventing hemorrhagic fever with renal syndrome [41].

The strengths of this study is the use of georeferenced address of each individual case (only $0.1 \%$ of the addresses were not be able to retrieved) which offered more geographic precision than studies based on administrative boundaries such as local government area, postal area or CCD. Using SaTScan enables us to analyse variations in any event of interest for many possible small and large geographic groups and without being restricted by administrative boundaries. In SaTScan, the shape of the scan window can be circular or elliptical. Circular windows provide good level of accuracy if the population at risk exists in circular shaped areas [29,50,51]. However, as population at risk do not exist in circular shaped areas, elliptical-shaped scan windows will provide slightly increased power for identifying non-circular shaped clusters, for example, long narrow clusters [52]. Tango and Takahashi recently proposed using flexible shaped spatial scan statistics to accommodate irregular shaped clusters
[51]. Another advantage is that where point data are not available, the centroids of the smallest geographical spatial unit that are available can be used in SaTScan [52]. Lastly, SaTScan is also capable of performing space-time scan statistics to identify clusters existing in both space and time [34].

However, there are also a number of limitations of using SaTScan to identify spatial clusters. For those wishing to explore modelling based approaches, SaTScan only implements scan statistic methods. Also, whilst it can identify clusters, it cannot explain why the variations in the risks of the events of interest exist. SaTScan also lacks an interface to other graphing, mapping and statistical packages. Another limitation of SaTScan is that it does not provide guidance for choosing the maximum size of the scan windows [40]. We were also unable to link multiple births to a mother and therefore could not account for this in our analyses. This could underestimate the standard error and overestimate the significance of socio-demographic characteristics. Further, we were not able to include all potential predictors or confounders of adverse maternal -related behaviours in our models as they were not available in our dataset. 
Nevertheless, geospatial analytical techniques are useful tools for identifying geographic areas for intervention. However, the usefulness of these geospatial techniques is only as good as the quality of the data that are available. Finally, although not a limitation of the study, an important point to bear in mind is that due to the large number of women in our study, small differences between women in the spatial clusters and women outside of the spatial clusters were likely to be detected as statistically significant in the descriptive analysis. The epidemiological and clinical importance of such small differences should also be considered in decision making rather than simply relying on statistical significance in informing decisions.

\section{Conclusion}

This study provides a first attempt, as far as we are aware, to visually and quantitatively identify and describe the spatial characteristics of ante-natal risk factors. We have demonstrated that using existing georeferenced routinely collected health data, GIS and geospatial techniques can be useful tools to assist policy makers to implement targeted interventions in smaller geographic areas and which will also ensure that limited resources are used efficiently.

\section{Competing interests}

The authors declare that they have no competing interests.

\section{Authors' contributions}

SC conducted the literature search, the analyses and drafted the manuscript. MN assisted with the analyses and provided expert advice on geospatial statistics. BJ, EH and JE conceptualised the ideas for this manuscript. All authors contributed to, and approved, the final manuscript.

\section{Acknowledgements}

The authors would like thank the Centre for Epidemiology and Evidence, New South Wales Ministry of Health for providing the birth data for the study and for approval to publish the manuscript.

\section{Author details \\ ${ }^{1}$ Centre for Research, Evidence Management and Surveillance, South Western Sydney Local Health Districts, Sydney, Australia. ${ }^{2}$ South Western Sydney Clinical School, University of New South Wales, Sydney, Australia. ${ }^{3}$ NSW Biostatistical Officer Training Program, NSW Ministry of Health, Sydney, Australia. ${ }^{4}$ Research Centre for Primary Health Care and Equity, University of New South Wales, Sydney, Australia. ${ }^{5}$ South Western Sydney Local Area Health District, Sydney, Australia. ${ }^{6}$ School of Public Health and Community Medicine, University of New South Wales, Sydney, Australia. ${ }^{7}$ School of Women's and Children's Health, University of New South Wales, Sydney, Australia. ${ }^{8}$ School of Public Health, University of Sydney, Sydney, Australia.}

Received: 22 July 2013 Accepted: 18 October 2013

Published: 24 October 2013

\section{References}

1. Neufeld MD, Frigon C, Graham AS, Mueller BA: Maternal infection and risk of cerebral palsy in term and preterm infants. J Perinatol 2005, 25:108-113.

2. Allen VM, Joseph KS, Murphy KE, Magee LA, Ohlsson A: The effect of hypertensive disorders in pregnancy on small for gestational age and stillbirth: a population based study. BMC Pregnancy Childbirth 2004, 4:1-8.
3. von Katterfeld B, Li J, McNamara B, Langridge AT: Maternal and neonatal outcomes associated with gestational diabetes in women from culturally and linguistically diverse backgrounds in Western Australia. Diabet Med 2012, 29:372-377.

4. Olausson PO, Cnattingius S, Haglund B: Teenage pregnancies and risk of late fetal death and infant mortality. BJOG 1999, 106:116-121.

5. Lumley J: How important is social class a factor in preterm birth? Lancet 1997, 349:1040-1041.

6. Australian Government. Department of Health and Ageing: Clinical practice guidelines. Antenatal care - Module 1; 2012. http://www.health.gov.au/ internet/publications/publishing.nsf/Content/clinical-practice-guidelines-acmod1.

7. National Institute for Health and Clinical Excellence: Antenatal care: Routine care for healthy pregnant women; 2003. http://www.guideline.gov/ content.aspx?id=14306.

8. Institute of Medicine: Preventing low birth weight. Washington, DC: National Academy Press; 1985.

9. Gortmacher SL: The effects of prenatal care upon the health of the newborn. Am J Public Health 1979, 69:653-657.

10. Moore TR, Origel W, Key TC, Resnik R: The perinatal and economic impact of prenatal care in a low-socioeconomic population. Am J Obstet Gynecol 1986, 154:29-33.

11. Goldenberg RL, Culhane JF: Low birth weight in the United States. Am Soc Clin Nutr 2007, 85:5845-5905.

12. Liu GG: Birth outcomes and the effectiveness of prenatal care. Health Serv Res 1998, 32:805-823.

13. Conway KS, Kennedy LD: Maternal depression and the production of infant health. South Econ J 2004, 71:260-286.

14. Herbst MA, Mercer BM, Beazley D, Meyer N, Carr T: Relationship of prenatal care and perinatal morbidity in low-birth-weight infants. Am J Obstet Gynecol 2003, 189:930-933.

15. McDonald TP, Coburn AF: Predictors of prenatal care utilization. Soc Sci Med 1988, 27:167-171.

16. LaVeist TA, Keith VM, Gutierrez ML: Black/white differences in prenatal care utilization: an assessment of predisposing and enabling factors. Health Serv Res 1995, 30:43-58.

17. Lieu TT, Rubin G: Late entry to antenatal care in New South Wales, Australia. Reprod Health 2006, 3:1-8.

18. Census US: Census 2000 Gateway. http://www.census.gov/main/www/ cen2000.html.

19. Eades S: Maternal and child health care services: actions in the primary health care setting to improve the health of aboriginal and Torres Strait Islander women of childbearing age. MEnzies School of Health Research: Infants and Young Children; 2004.

20. US Department of Health and Human Services: The health consequences of smoking. A report of the Surgeon General. Rockville: MD: US Department of Health and Human Services, Public Health Service, Office of the Surgeon General; 2004

21. CDC: State estimates of neonatal health-care costs associated with maternal smoking - United States, 1996. MMWR 2004, 53:911-915.

22. Linacre S: Australian social trends 2007. Australian Bureau of Statistics: Australia's Babies; 2007.

23. Mohsin M, Bauman AE: Socio-demographic factors associated with smoking and smoking cessation among 426,366 pregnant women in New South Wales, Australia. BMC Public Health 2005, 5:1-9.

24. Newnham J: 12th report of the perinatal and infant mortality committee of Western Australia. Western Australia: Department of Health; 2007.

25. Olds D, Hendenson CR, Chamberlin R, Tatelbaum RC: Preventing child abuse and neglect: a randomized trial of nurse home visitation. Pediatrics 1986, 78:65-78.

26. Olds D, Hendenson CR, Kitzman HJ, Eckenrode JJ, Cole RE, Tatelbaum RC: Prenatal and infancy home visitation by nurses: recent findings. Future Child 1999, 9:44-65.

27. Odoi A, Martin SW, Michel P, Middleton D, Holt J, Wilson J: Investigation of clusters of giardiasis using GIS and a spatial scan statistic. Int $J$ Health Geogr 2004, 3:11.

28. Fukuda Y, Umezaki M, Nakamura K, Takano T: Variations in societal characteristics of spatial disease clusters: examples of colon, lung and breast cancer in Japan. Int J Health Geogr 2005, 4:1-13.

29. Warden C: Comparison of poisson and Bernoulli spatial cluster analyses of pediatric injuries in a fire district. Int J Health Geogr 2008, 7:1-17. 
30. Pollack LA, Gotway CA, Bates JH, Parikh-Patel A, Richards TB, Seeff LC, Hodges H, Kassim S: Use of the spatial scan statistic to identify geographic variations in late stage colorectal cancer in California (United States). Cancer Causes Control 2006, 17:449-457.

31. ABS: 2011 Census QuickStats. Australia: ABS; 2013.

32. ABS: 2001 Census of Population and Housing - Geographic Areas; 2006. http://www.abs.gov.au/websitedbs/D3110124.nsf/f5c7b8fb229cf017ca 256973001fecec/a17dc48d988ecf63ca256dad00005ea3!OpenDocument.

33. ABS: Census of population and housing: socio-economic indexes for areas (SEIFA), Australia. Canberra, Australia: ABS cat. no. 20330.0.55.001; 2006.

34. Kulldorff M: SaTScan v9.1. Software for the Spatial and Space-Time Scan Statistics. Computer Software; 2011.

35. Kulldorff M: A spatial scan statistic. Commun Stat: Theory and Methods 1997, 26:1481-1496.

36. Kulldorff M: Prospective time-periodic geographic disease surveillance using a scan statistic. J R Stat Soc 1997, 164:61-72.

37. RStudio: RStudio: Integrated development environment for $R$ (Version 0.96. 122). Boston, MA: RStudio; 2012.

38. Coleman M, Coleman M, Mabuza A, Kok G, Coetzee M, Durrheim D: Using the SaTScan method to detect local malaria clusters for guiding malaria control programmes. Malar J 2009, 8:68.

39. Green C, Hoppa RD, Young TK, Blanchard JF: Geographic analysis of diabetes prevalence in an urban area. Soc Sci Med 2003, 57:551-560.

40. Chen J, Roth RE, Naito AT, Lengerich EJ, MacEachren AM: Geovisual analytics to enhance spatial scan statistic interpretation: an analysis of U.S. cervical cancer mortality. Int J Health Geogr 2008, 7:1-18.

41. Fang LYL, Liang S, Vlas S, Feng D, Han X, Zhao W, Xu B, Bian L, Yang H, Gong P, Richardus JH, Cao W: Spatial analysis of hemorrhagic fever with renal syndrome in China. BMC Infect Dis 2006, 6:6.

42. Rothman KJ: A sobering start for the cluster busters' conference. Am J Epidemiol 1990, 132:6-13.

43. Perloff JD, Jaffee KD: Late entry into prenatal care: the neighborhood context. Soc Work 1999, 44:116-128.

44. Magadi MA, Madise NJ, Rodrigues RN: Frequency and timing of antenatal care in Kenya: explaining the variations between women of different communities. Soc Sci Med 2000, 51:551-561.

45. Carter R, Mendis KN, Roberts D: Spatial targeting of interventions against malaria. Bull World Health Org 2000, 78:1401-1411.

46. Kuehl KS, Loffredo CA: A cluster of hypoplastic left heart malformation in Baltimore, Maryland. Pediatr Cardiol 2006, 27:25-31.

47. Wang F, Xue F, Chen Y, Ma Y, Liu Y: The spatial epidemiology of tuberculosis in Linyi City, China, 2005-2010. BMC Public Health 2012, 12:1-8.

48. Chen J: Visual analytics of spatial scan statistic results. Utah: In Geospatial Visual Analytics Workshop, GIScience conference; 2008.

49. Huang L, Stinchcomb DG, Pickle LW, Dill J, Berrigan D: Identifying clusters of active transportation using Spatial Scan Statistics. Am J Prev Med 2009, 37:157-166.

50. Patil GPTC: Geographic and network surveillance via scan statistics for critical area detection. Stat Sci 2003, 18:457-465.

51. Tango T, Takahashi $\mathrm{K}:$ A flexibly shaped spatial scan statistisc for detecting clusters. Int I Health Geogr 2005, 4:1-15.

52. Kulldorff M: SatScan user guide; 2010. http://www.satscan.org.

doi:10.1186/1476-072X-12-46

Cite this article as: Chong et al.: Geospatial analyses to identify clusters of adverse antenatal factors for targeted interventions. International Journal of Health Geographics 2013 12:46.

\section{Submit your next manuscript to BioMed Central and take full advantage of:}

- Convenient online submission

- Thorough peer review

- No space constraints or color figure charges

- Immediate publication on acceptance

- Inclusion in PubMed, CAS, Scopus and Google Scholar

- Research which is freely available for redistribution

Submit your manuscript at www.biomedcentral.com/submit
Ciomed Central 